\title{
Data Clarification
}

National Cancer Institute

\section{Source}

National Cancer Institute. Data Clarification. NCI Thesaurus. Code C142471.

The explanation or answer supplied in response to a data query. 